\title{
Transient Solution of Two-Dimensional State M/G/1 Queuing Model with Working Vacations
}

\author{
Indra \\ Associate Professor \\ Department of Statistics \& Operational Research, \\ Kurukshetra University Kurukshetra, \\ Haryana, INDIA
}

\author{
Ruchi \\ Research Scholar \\ Department of Statistics \& Operational Research, \\ Kurukshetra University Kurukshetra, \\ Haryana, INDIA
}

\begin{abstract}
Transient behavior of a first-come first-served, twodimensional state $\mathrm{M} / \mathrm{G} / 1$ queuing model with working vacation is studied. As soon as the system becomes empty, the server leaves the system and takes vacation for random duration during which it may perform ancillary duty and is called on working vacation. The server works with different service rate rather than completely stopping service during a vacation. Both service times during busy period and vacation period and vacation times are assumed to follow general distribution. The Laplace transform of the probabilities of exact number of arrivals and departure by a given time are obtained. The emphasis in this paper is theoretical but numerical assessment of operational consequences is also given and presented graphically. Finally some particular cases are derived there from.
\end{abstract}

\section{Keywords}

M/G/1 queue; Working vacation; Two-dimensional state model; Laplace Transform

\section{INTRODUCTION}

Much of the vast literature on queueing models with working vacation is confined to results describing steady-state operation only. But in many potential applications of queueing theory, the practitioner needs to know how the system will operate up to some instant t. Furthermore, if the system is empty initially, the fraction of time the server is busy and the initial rate of output, etc. will be below the steady-state values, and hence, the use of steady-state results to obtain these measures is not appropriate. Thus, the investigation of the transient behaviour of queueing processes is also important from the point of view of the theory and its applications.

In the present paper, we consider an $\mathrm{M} / \mathrm{G} / 1$ queueing model with Exhaustive service and Multiple vacations such that the server works with different service rates rather than completely stops service during a vacation period. Such a vacation is called 'Working Vacation'. The server begins a working vacation of random length at the instant when the queue becomes empty. If the server returns from a working vacation to find the system not empty, it starts to work immediately and continues until the system becomes empty again (Exhaustive service). If the server returns from a working vacation to find no customers waiting, it begins another working vacation immediately, and continues in this manner until it finds at least one customer waiting upon returning from a working vacation (Multiple working vacations). It was Servi \& Finn [10] who first analyzed the queueing system with working vacations and obtained the queue length distribution, mean and variance of the $\mathrm{M} / \mathrm{M} / 1 / \mathrm{WV}$ queue. Subsequently, Wu \& Takagi [11] generalized the result of Servi \& Finn [10] to an M/G/1 queue with working vacations. He derived the distributions for the queue size and the system time for an arbitrary customer in the steady state. Many researchers like Kim, Choi \& Chae [6], Baba [1], Banik et al. [2], Liu et al. [8] and Li \& Tian [7] worked on queueing system with working vacations.

\section{MODEL DESCRIPTION}

In this paper, we analyze the transient behavior of two state $\mathrm{M} / \mathrm{G} / 1$ queue with working vacations. The state of the system is given by $(i, j)$, where ' $i$ ' is the number of arrivals and ' $j$ ' is the number of departures until time $t$. This queueing system is referred to as the two state $\mathrm{M} / \mathrm{G} / 1 / \mathrm{WV}$ queue in this paper. We follow Pegden \& Rosenshine [9], and obtained a more informative measure by finding out

$>$ Laplace transforms of the probabilities of exact number of arrivals and departures by a given time

$>$ Number of units arrived by time $t$

$>$ Number of units served by time $t$

$>$ Waiting time distribution and cumulative distribution for sojourn time

$>$ Server's utilization time

numerically and graphically for the above situations for the case when the service times and vacation times are exponential. Finally some particular cases of interest are derived there from.

The queueing system investigated in this paper assumes that during the working vacation period and otherwise also the customers arrive according to a poisson process with rate $\lambda$. Queue discipline is first-come-first-served. The service time distribution during busy period and working vacation period is general with probability density $\mathrm{D} 1(\mathrm{x})$ and $\mathrm{D} 2(\mathrm{y})$ respectively. Working vacation time distribution is general with probability density D3(y). Various stochastic processes involved in the system are statistically independent.

\section{DEFINITIONS AND NOTATIONS}

$\mathrm{P}_{\mathrm{i}, \mathrm{j}, \mathrm{B}}(\mathrm{x}, \mathrm{t}) \Delta=$ The probability that there are exactly $\mathrm{i}$ arrivals and $\mathrm{j}$ departures by time $\mathrm{t}$ and the server is busy in relation to the queue and the elapsed service time lies between $\mathrm{x}$ and $\mathrm{X}+\Delta, \mathrm{i}>\mathrm{j}$

$\mathrm{P}_{\mathrm{i}, \mathrm{j}, \mathrm{V}}(\mathrm{y}, \mathrm{t}) \Delta=$ The probability that there are exactly $\mathrm{i}$ arrivals and $\mathrm{j}$ departures by time $\mathrm{t}$ and the server is on working vacation and the elapsed service time during vacation lies between $y$ and $y+\Delta, i \geq j$ 

departures by time $\mathrm{t}$.

$\eta_{1}(\mathrm{x}) \Delta, \eta_{2}(\mathrm{y}) \Delta$ and $\eta_{3}(\mathrm{y}) \Delta$ are the first order probabilities that the corresponding service time during busy period, service time during vacation period and vacation time will be completed in time respectively provided the same had not been completed till time $\mathrm{x}$ and $\mathrm{y}$. And

$D_{1}(x)=\eta_{1}(x) e^{-\int_{0}^{x} \eta_{1}(u) d u}, D_{2}(y)=\eta_{2}(y) e^{-\int_{0}^{y} \eta_{2}(v) d v}$

and $D_{3}(y)=\eta_{3}(y) e^{-\int^{y} \eta_{3}(v) d v}$

The Laplace transform of $P_{i, j}(t)$

$\overline{\mathrm{P}}_{\mathrm{i}, \mathrm{j}}(\mathrm{s})=\int_{0}^{\infty} \mathrm{e}^{-\mathrm{st}} \mathrm{P}_{\mathrm{i}, \mathrm{j}}(\mathrm{t}) \mathrm{dt} \operatorname{Re}(\mathrm{s})>0$

\section{SOLUTION OF THE PROBLEM}

Initially

$$
\mathrm{P}_{0,0, \mathrm{~V}}(0,0)=1
$$

\& clearly

$$
\mathrm{P}_{0,0, \mathrm{~B}}(0,0)=0
$$

The difference-differential equations governing the$$
\frac{\partial}{\partial t} P_{i, j, B}(x, t)+\frac{\partial}{\partial x} P_{i, j, B}(x, t)=-\left\{\lambda+\eta_{1}(x)\right\} P_{i, j, B}(x, t)+\lambda P_{i-1,}
$$$$
\mathrm{i}>\mathrm{j} \geq 0
$$

$P_{i, j}(t)=$ The probability that there are exactly $i$ arrivals and $j$

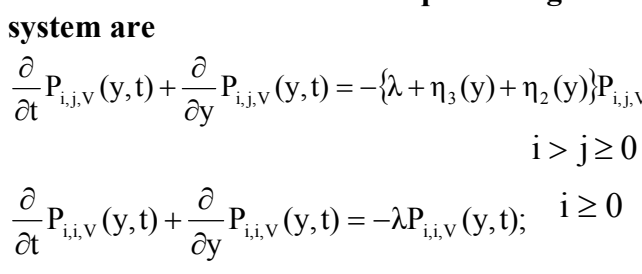

$$
\begin{aligned}
& \overline{\mathrm{P}}_{\mathrm{i}, 0, \mathrm{~B}}(\mathrm{~s})=\int_{0}^{\infty} \mathrm{e}^{-\int_{0}^{\mathrm{x}}\left(\mathrm{s}+\lambda+\eta_{1}(\mathrm{u}) \mathrm{du}\right.} \sum_{\mathrm{k}_{1}=0}^{\mathrm{i}-1}\left(\prod_{\mathrm{k}_{2}=1}^{\mathrm{i}-1-\mathrm{k}_{1}} \int_{0}^{\mathrm{x}_{\mathrm{k}_{2}}}\right) \lambda^{\mathrm{i}-1-\mathrm{k}_{1}} \int_{0}^{\infty} \eta_{3}(\mathrm{y}) \lambda^{\mathrm{k}_{1}+1} \\
& e^{-\int^{y}\left(s+\lambda+\eta_{3}(v)+\eta_{2}(v)\right) d v}\left(\prod_{k_{2}=1}^{k_{1}+1} \int_{0}^{y_{k_{2}}}\right) e^{\int_{0}^{y}\left(\eta_{3}(v)+\eta_{2}(v)\right) d v}\left(\prod_{k_{2}=1}^{k_{1}+1} d y_{k_{1}+2-k_{2}}\right) d y \\
& \left(\prod_{\mathrm{k}_{2}=1}^{\mathrm{i}-1-\mathrm{k}_{1}} \mathrm{dx}_{\mathrm{i}-\mathrm{k}_{2}-\mathrm{k}_{1}}\right) \mathrm{dx} \quad ; \mathrm{i}>0
\end{aligned}
$$

$\overline{\mathrm{P}}_{\mathrm{i}, \mathrm{j}, \mathrm{V}}(\mathrm{s})=\int_{0}^{\infty} \mathrm{e}^{-\int_{0}^{\mathrm{y}}\left(\mathrm{s}+\lambda+\eta_{3}(\mathrm{v})+\eta_{2}(\mathrm{v})\right) \mathrm{dv}}\left\{\sum_{\mathrm{k}_{1}=0}^{\mathrm{i}-\mathrm{j}} \lambda^{\mathrm{i}-\mathrm{j}-\mathrm{k}_{1}}\left(\prod_{\mathrm{k}_{2}=1}^{\mathrm{i}-\mathrm{j}-\mathrm{k}_{1}} \int_{0}^{\mathrm{y}_{\mathrm{k}_{2}}}\right)\right.$

$\int_{0}^{\infty} \eta_{2}(y) \bar{P}_{k_{1}+j, j-1, v}(y, s) d y\left(e^{\int^{y}\left(\eta_{3}(v)+\eta_{2}(v)\right) d v}\right)^{\delta_{k_{1}, 0}}$

$$
\left(\prod_{k_{2}=1}^{i-j-k_{1}} d y_{i-j-k_{1}-k_{2}}\right)+\lambda^{i-j}\left(\prod_{k_{1}=1}^{i-j} \int_{0}^{y_{k_{1}}} \int_{0}^{\infty} \eta_{1}(x) \bar{P}_{j, j-1, B}(x, s) d x\right.
$$$$
\left.\mathrm{e}^{\int^{\mathrm{y}}\left(\eta_{3}(\mathrm{v})+\eta_{2}(\mathrm{v})\right) \mathrm{dv}}\left(\prod_{\mathrm{k}_{1}=1}^{\mathrm{i}-\mathrm{j}} \mathrm{dy} \mathrm{y}_{\mathrm{i}-\mathrm{j}-\mathrm{k}_{1}}\right)\right\} \mathrm{dy}
$$

$$
; \mathrm{i}>\mathrm{j} \geq 1
$$

The appropriate boundary conditions are

$$
\begin{aligned}
& P_{i, j, V}(0, t) \Delta=\int_{0}^{\infty} \eta_{2}(y) P_{i, j-1, V}(y, t) d y\left(1-\delta_{j, 0}\right) \\
& P_{i, i, V}(0, t) \Delta=\int_{0}^{\infty} \eta_{2}(y) P_{i, i-1, V}(y, t) d y+\int_{0}^{\infty} \eta_{1}(x) P_{i, i-1, B}(x, t) d x \\
& P_{i, j, B}(0, t) \Delta=\int_{0}^{\infty} \eta_{3}(y) P_{i, j, V}(y, t) d y+\int_{0}^{\infty} \eta_{1}(x) P_{i, j-1, B}(x, t) d x\left(1-\delta_{j, 0}\right) \\
& i>j \geq 0
\end{aligned}
$$

Using (1) in equations (4) to (6) along with initial conditions and solving recursively, we have

$$
\begin{aligned}
& \overline{\mathrm{P}}_{0,0, \mathrm{~V}}(\mathrm{~s})=\frac{1}{\mathrm{~s}+\lambda}
\end{aligned}
$$

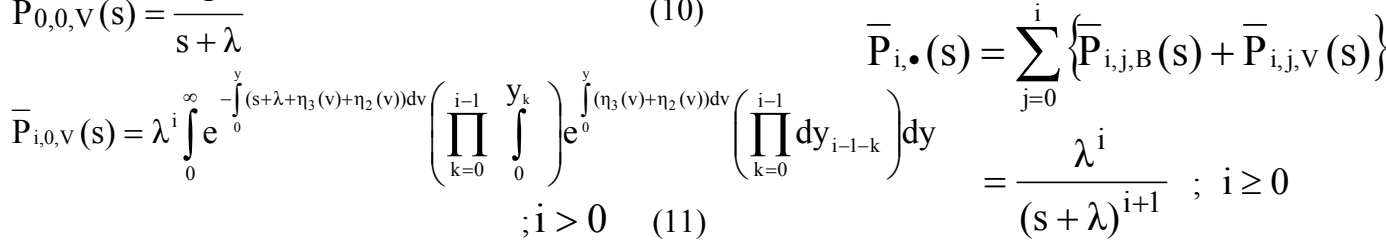

\section{VERIFICATION OF MODEL}

The Laplace transform $\overline{\mathrm{P}}_{\mathrm{i}, \bullet}(\mathrm{s})$ of the probability $\mathrm{P}_{\mathrm{i}, \boldsymbol{\bullet}}(\mathrm{t})$ that 
Clearly $\sum_{\mathrm{i}=0}^{\infty} \sum_{\mathrm{j}=0}^{\mathrm{i}}\left\{\overline{\mathrm{P}}_{\mathrm{i}, \mathrm{j}, \mathrm{V}}(\mathrm{s})+\overline{\mathrm{P}}_{\mathrm{i}, \mathrm{j}, \mathrm{B}}(\mathrm{s})\right\}=\frac{1}{\mathrm{~s}}$

for $\quad \sum_{i=0}^{\infty} \overline{\mathrm{P}}_{\mathrm{i}, \bullet}(\mathrm{s})=\frac{1}{\mathrm{~s}}$

or $\quad \sum_{i=0}^{\infty} \mathrm{P}_{\mathrm{i}, \bullet}(\mathrm{t})=1$, a verification.

The Laplace transform of the mean number of arrivals by time $\mathrm{t}$, is

$$
\sum_{i=0}^{\infty} i \bar{P}_{i, \bullet}(s)=\frac{\lambda}{s^{2}}
$$

Hence mean number of arrivals by time $t=\lambda t$, an obvious result.

The Laplace Transform $\bar{P}_{\bullet}, j(s)$ of the probability $P_{\bullet}, j(t)$ that exactly $\mathrm{j}$ units depart by time $\mathrm{t}$ is

$\overline{\mathrm{P}} \bullet, \mathrm{j}(\mathrm{s})=\sum_{\mathrm{i}=\mathrm{j}}^{\infty}\left(\overline{\mathrm{P}}_{\mathrm{i}, \mathrm{j}, \mathrm{V}}(\mathrm{s})+\overline{\mathrm{P}}_{\mathrm{i}, \mathrm{j}, \mathrm{B}}(\mathrm{s})\right)$

\section{PARTICULAR CASES}

In this section, we shall examine whether by setting appropriate parameters, our results are consistent with known results for some specific cases.

Case I- Results for the case, when the service time during busy period, service time during vacation period and vacation time are exponentially distributed i.e. $\eta_{1}(x)=\mu_{B}, \eta_{2}(y)=\mu_{v}$, and $\eta_{3}(y)=w$ are obtained by substituting $\quad \eta_{1}(\mathrm{x})=\mu_{\mathrm{B}}, \eta_{2}(\mathrm{y})=\mu_{\mathrm{v}}$ and $\eta_{3}(\mathrm{y})=\mathrm{w}$ in equations (10) to (15).

$\overline{\mathrm{P}}_{0,0, \mathrm{~V}}(\mathrm{~s})=\frac{1}{\mathrm{~s}+\lambda}$

$\overline{\mathrm{P}}_{\mathrm{i}, 0, \mathrm{~V}}(\mathrm{~s})=\frac{\lambda^{\mathrm{i}}}{(\mathrm{s}+\lambda)\left(\mathrm{s}+\lambda+\mu_{\mathrm{V}}+\mathrm{w}\right)^{\mathrm{i}}} ; \mathrm{i}>0$

$\overline{\mathrm{P}}_{\mathrm{i}, 0, \mathrm{~B}}(\mathrm{~s})=\frac{\lambda^{\mathrm{i}} \mathrm{w}}{(\mathrm{s}+\lambda)\left(\mathrm{s}+\lambda+\mu_{\mathrm{B}}\right)\left(\mathrm{s}+\lambda+\mu_{\mathrm{v}}+\mathrm{w}\right)} \sum_{\mathrm{k}=0}^{\mathrm{i}-1}\left(\frac{1}{\mathrm{~s}+\lambda+\mu_{\mathrm{B}}}\right)^{\mathrm{k}}$ $\left(\frac{1}{\mathrm{~s}+\lambda+\mu_{\mathrm{v}}+\mathrm{w}}\right)^{\mathrm{i}-\mathrm{k}-1} \quad ; \mathrm{i}>0$

$\overline{\mathrm{P}}_{\mathrm{i}, \mathrm{i}, \mathrm{V}}(\mathrm{s})=\left(\frac{1}{\mathrm{~s}+\lambda}\right)\left(\mu_{\mathrm{V}} \overline{\mathrm{P}}_{\mathrm{i}, \mathrm{i}-1, \mathrm{~V}}(\mathrm{~s})+\mu_{\mathrm{B}} \overline{\mathrm{P}}_{\mathrm{i}, \mathrm{i}-1, \mathrm{~B}}(\mathrm{~s})\right)$

$; \mathrm{i}>0$

$\overline{\mathrm{P}}_{\mathrm{i}, \mathrm{j}, \mathrm{V}}(\mathrm{s})=\int_{0}^{\infty} \mathrm{e}^{-\left(\mathrm{s}+\lambda+\mu_{\mathrm{V}}+\mathrm{w}\right) \mathrm{y}}\left\{\sum_{\mathrm{k}_{1}=0}^{\mathrm{i}-\mathrm{j}} \lambda^{\mathrm{i}-\mathrm{j}-\mathrm{k}_{1}}\left(\prod_{\mathrm{k}_{2}=1}^{\mathrm{i}-\mathrm{j}-\mathrm{k}_{1}} \int_{0}^{\mathrm{y}_{\mathrm{k}_{2}}}\right) \mu_{\mathrm{V}}\right.$

$\overline{\mathbf{P}}_{\mathrm{k}_{1}+\mathrm{j}, \mathrm{j}-1, \mathrm{v}}(\mathrm{s})\left(\mathrm{e}^{\left(\mu_{\mathrm{V}}+\mathrm{w}\right) \mathrm{y}}\right)^{\delta_{\mathrm{k}_{1}, 0}}\left(\prod_{\mathrm{k}_{2}=1}^{\mathrm{i}-\mathrm{j}-\mathrm{k}_{1}} \mathrm{dy}_{\mathrm{i}-\mathrm{j}-1-\mathrm{k}_{1}-\mathrm{k}_{2}}\right)+$

$\left.\lambda^{i-j}\left(\prod_{k_{1}=1}^{i-j} \int_{0}^{y_{k_{1}}}\right) \mu_{B} \bar{P}_{j, j-1, B}(s) e^{\left(\mu_{v}+w\right) y}\left(\prod_{k_{1}=1}^{i-j} d y_{i-j-k_{1}}\right)\right\} d y$

$; \mathrm{i}>\mathrm{j} \geq 1$

$$
\begin{aligned}
& \bar{P}_{i, j, B}(s)=\int_{0}^{\infty} e^{-\left(s+\lambda+\mu_{B}\right) x}\left\{\sum_{k_{1}=0}^{i-j-1}\left(\prod_{k_{2}=1}^{i-j-k_{1}} \int_{0}^{x_{k_{2}}}\right) \lambda^{i-j-1-k_{1}} W\right. \\
& \bar{P}_{k_{1}+j+1, j, V}(s)\left(\prod_{k_{2}=1}^{i-j-1-k_{1}} d^{i-j-k_{1}-k_{2}}\right)+\sum_{k_{1}=0}^{i-j-1}\left(\prod_{k_{2}=1}^{i-j-1-k_{1}} \int_{0}^{x_{k_{2}}}\right) \mu_{B} \\
& \left.\lambda^{i-j-1-k_{1}} \bar{P}_{k_{1}+j+1, j-1, B}(s)\left(\prod_{k_{2}=1}^{i-j-1-k_{1}} d x_{i-j-k_{1}-k_{2}}\right)\right\} d x
\end{aligned}
$$

(20) to (25) coincide with corresponding equations (10) to (13) of Indra \& Ruchi [5].

\section{SUB-CASES}

Sub-case I(a) - When there is no work during vacation period i.e. $\mu_{\mathrm{V}}$ is zero. Letting $\mu_{\mathrm{V}}=0$ in equations (20) to (25), we have

$$
\begin{aligned}
& \overline{\mathrm{P}}_{0,0, \mathrm{~V}}(\mathrm{~s})=\frac{1}{\mathrm{~s}+\lambda} \\
& \overline{\mathrm{P}}_{\mathrm{i}, 0, \mathrm{~V}}(\mathrm{~s})=\frac{\lambda^{\mathrm{i}}}{(\mathrm{s}+\lambda)(\mathrm{s}+\lambda+\mathrm{w})^{\mathrm{i}}} ; \mathrm{i}>0 \\
& \overline{\mathrm{P}}_{\mathrm{i}, 0, \mathrm{~B}}(\mathrm{~s})=\frac{\lambda^{\mathrm{i}} \mathrm{w}}{(\mathrm{s}+\lambda)(\mathrm{s}+\lambda+\mu)(\mathrm{s}+\lambda+\mathrm{w})} \sum_{\mathrm{k}=0}^{\mathrm{i}-1}\left(\frac{1}{\mathrm{~s}+\lambda+\mu}\right)^{\mathrm{k}} \\
& \left(\frac{1}{\mathrm{~s}+\lambda+\mathrm{w}}\right)^{\mathrm{i}-\mathrm{k}-1} \quad ; \mathrm{i}>0
\end{aligned}
$$

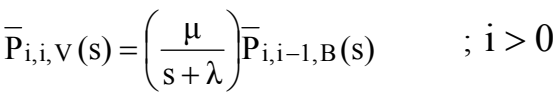

$$
\begin{aligned}
& \left.\overline{\mathrm{P}}_{\mathrm{i}, \mathrm{j}, \mathrm{V}}(\mathrm{s})=\int_{0}^{\infty} \mathrm{e}^{-(\mathrm{s}+\lambda+w) y} \lambda^{i-j}\left(\prod_{\mathrm{k}_{1}=1}^{\mathrm{i}-\mathrm{j}} \int_{0}^{\mathrm{y}_{\mathrm{k}_{1}}}\right) \mu \overline{\mathrm{P}}_{\mathrm{j}, \mathrm{j}-1, \mathrm{~B}}(\mathrm{~s}) \mathrm{e}^{\mathrm{wy}}\left(\prod_{\mathrm{k}_{1}=1}^{\mathrm{i}-\mathrm{j}} \mathrm{dy} \mathrm{y}_{\mathrm{i}-\mathrm{j}-\mathrm{k}_{1}}\right)\right\} d y \\
& ; \mathrm{i}>\mathrm{j} \geq 1 \\
& \overline{\mathrm{P}}_{\mathrm{i}, \mathrm{j}, \mathrm{B}}(\mathrm{s})=\int_{0}^{\infty} \mathrm{e}^{-(\mathrm{s}+\lambda+\mu) \mathrm{x}}\left\{\sum_{\mathrm{k}_{1}=0}^{\mathrm{i}-\mathrm{j}-1}\left(\prod_{\mathrm{k}_{2}=1}^{\mathrm{i}-\mathrm{j}-\mathrm{k}_{1}} \int_{0}^{\mathrm{X}_{\mathrm{k}_{2}}}\right) \lambda^{\mathrm{i}-\mathrm{j}-1-\mathrm{k}_{1}} w \overline{\mathrm{P}}_{\mathrm{k}_{1}+\mathrm{j}+1, \mathrm{j}, \mathrm{V}}\right. \\
& \left(\prod_{\mathrm{k}_{2}=1}^{\mathrm{i}-\mathrm{j}-1-\mathrm{k}_{1}} \mathrm{dx} \mathrm{x}_{\mathrm{i}-\mathrm{j}-\mathrm{k}_{1}-\mathrm{k}_{2}}\right)+\sum_{\mathrm{k}_{1}=0}^{\mathrm{i}-\mathrm{j}-1}\left(\prod_{\mathrm{k}_{2}=1}^{\mathrm{i}-\mathrm{j}-1-\mathrm{k}_{1}} \int_{0}^{\mathrm{x}_{\mathrm{k}_{2}}}\right) \mu \lambda^{\mathrm{i}-\mathrm{j}-1-\mathrm{k}_{1}} \\
& \left.\overline{\mathrm{P}}_{\mathrm{k}_{1}+\mathrm{j}+1, \mathrm{j}-1, \mathrm{~B}}(\mathrm{~s})\left(\prod_{\mathrm{k}_{2}=1}^{\mathrm{i}-\mathrm{j}-1-\mathrm{k}_{1}} \mathrm{~d} \mathrm{x}_{\mathrm{i}-\mathrm{j}-\mathrm{k}_{1}-\mathrm{k}_{2}}\right)\right\} \mathrm{dx}
\end{aligned}
$$$$
; \mathrm{i}>\mathrm{j} \geq 1
$$

These results agree with equations (1.2.15) to (1.2.20) of Indra [4].

Sub-case I(b) - Along with case I (a), when server is instantaneously available and he does not go for a vacation, the mean vacation time $\mathrm{w}^{-1}=0$ in (26) to (31), we have

$$
\begin{aligned}
& \overline{\mathrm{P}}_{0,0}(\mathrm{~s})=\overline{\mathrm{P}}_{0,0, \mathrm{~V}}(\mathrm{~s})=\frac{1}{\mathrm{~s}+\lambda} \\
& \overline{\mathrm{P}}_{\mathrm{i}, 0}(\mathrm{~s})=\overline{\mathrm{P}}_{\mathrm{i}, 0, \mathrm{~B}}(\mathrm{~s})=\left(\frac{\lambda}{\mathrm{s}+\lambda+\mu}\right)^{\mathrm{i}} \frac{1}{\mathrm{~s}+\lambda} \quad ; \mathrm{i}>0
\end{aligned}
$$


$\overline{\mathrm{P}}_{\mathrm{i}, \mathrm{i}}(\mathrm{s})=\overline{\mathrm{P}}_{\mathrm{i}, \mathrm{i}, \mathrm{V}}(\mathrm{s})=\left(\frac{\mu}{\mathrm{s}+\lambda}\right) \overline{\mathrm{P}}_{\mathrm{i}, \mathrm{i}-1, \mathrm{~B}}(\mathrm{~s}) \quad ; \mathrm{i}>0$

$\overline{\mathrm{P}}_{\mathrm{i}, \mathrm{j}}(\mathrm{s})=\overline{\mathrm{P}}_{\mathrm{i}, \mathrm{j}, \mathrm{B}}(\mathrm{s})=\left(\frac{\lambda}{\mathrm{s}+\lambda+\mu}\right)^{\mathrm{i}}\left(\frac{\mu}{\mathrm{s}+\lambda}\right)^{\mathrm{j}} \sum_{\mathrm{k}=0}^{\mathrm{j}} \frac{(\mathrm{i}-\mathrm{k})(\mathrm{i}+\mathrm{k}-1) !}{\mathrm{k} ! \mathrm{i} !}$

$\frac{(\mathrm{s}+\lambda)^{\mathrm{k}-1}}{(\mathrm{~s}+\lambda+\mu)^{\mathrm{k}}} \quad ; \mathrm{i}>\mathrm{j}>0$

(32) to (35) coincide with equation (5) of Pegden \& Rosenshine [9].

Case II - When server is on vacation only, then rate of doing work during vacation period is zero. Letting $\eta_{2}(y)=0$ in (10) to (15), we have

$\overline{\mathrm{P}}_{0,0, \mathrm{~V}}(\mathrm{~s})=\frac{1}{\mathrm{~s}+\lambda}$

$\overline{\mathrm{P}}_{\mathrm{i}, 0, \mathrm{~V}}(\mathrm{~s})=\lambda^{\mathrm{i}} \int_{0}^{\infty} \mathrm{e}^{-\int_{0}^{\mathrm{y}}\left(\mathrm{s}+\lambda+\eta_{3}(\mathrm{w}) \mathrm{dw}\right.}\left(\prod_{\mathrm{k}=0}^{\mathrm{i}-1} \int_{0}^{y_{k}}\right) \mathrm{e}^{\int^{\mathrm{y}} \eta_{3}(\mathrm{w}) \mathrm{dw}}$

$\left(\prod_{k=0}^{i-1} d_{i-1-k}\right) d y$

$$
\text { ; i }>0 \quad \text { (37) }
$$

$\overline{\mathrm{P}}_{\mathrm{i}, 0, \mathrm{~B}}(\mathrm{~s})=\int_{0}^{\infty} \mathrm{e}^{-\int^{\mathrm{x}}\left(\mathrm{s}+\lambda+\eta_{1}(\mathrm{u}) \mathrm{du}\right.} \sum_{\mathrm{k}_{1}=0}^{\mathrm{i}-1}\left(\prod_{\mathrm{k}_{2}=1}^{\mathrm{i}-1-\mathrm{k}_{1}} \int_{0}^{\mathrm{x}_{\mathrm{k}_{2}}}\right) \lambda^{\mathrm{i}-1-\mathrm{k}_{1}} \int_{0}^{\infty} \eta_{3}(\mathrm{z}) \lambda^{\mathrm{k}+1}$

$\mathrm{e}^{-\int_{0}^{\mathrm{y}}\left(\mathrm{s}+\lambda+\eta_{3}(w)\right) d w}\left(\prod_{\mathrm{k}_{2}=1}^{\mathrm{k}_{1}+1} \int_{0}^{y_{k_{2}}}\right) \mathrm{e}^{\int_{0}^{\mathrm{y}}\left(\eta_{3}(w)\right) d w}\left(\prod_{k_{2}=1}^{k_{1}+1} d y_{k_{1}+2-k_{2}}\right)$

$\left(\prod_{\mathrm{k}_{2}=1}^{\mathrm{i}-1-\mathrm{k}_{1}} \mathrm{dx}_{\mathrm{i}-\mathrm{k}_{2}-\mathrm{k}_{1}}\right) \mathrm{dx} \quad ; \mathrm{i}>0$

$\overline{\mathrm{P}}_{\mathrm{i}, \mathrm{i}, \mathrm{V}}(\mathrm{s})=\int_{0}^{\infty} \mathrm{e}^{-(\mathrm{s}+\lambda) \mathrm{y}}\left\{\int_{0}^{\infty} \eta_{1}(\mathrm{x}) \overline{\mathrm{P}}_{\mathrm{i}, \mathrm{i}-1, \mathrm{~B}}(\mathrm{x}, \mathrm{s}) \mathrm{dx}\right\} \mathrm{dy}$

$; \mathrm{i}>0$

$\overline{\mathrm{P}}_{\mathrm{i}, \mathrm{j}, \mathrm{V}}(\mathrm{s})=\int_{0}^{\infty} \mathrm{e}^{-\int^{\mathrm{y}}\left(\mathrm{s}+\lambda+\eta_{3}(\mathrm{w}) \mathrm{dw}\right.} \lambda^{\mathrm{i}-\mathrm{j}}\left(\prod_{\mathrm{k}_{1}=1}^{\mathrm{i}-\mathrm{j}} \int_{0}^{\mathrm{y}_{\mathrm{k}_{1}}} \int_{0}^{\infty} \eta_{1}^{\infty} \eta_{1}(\mathrm{x}) \overline{\mathrm{P}}_{\mathrm{j}, \mathrm{j}-1, \mathrm{~B}}(\mathrm{x}, \mathrm{s}) \mathrm{dx}\right.$

$\mathrm{e}^{\int^{y}\left(n_{3}(w) d w\right.} *\left(\prod_{k_{1}=1}^{i-j} d y_{i-j-k_{1}}\right) d y$

$; \mathrm{i}>\mathrm{j} \geq 1$

$1(40)$

$$
; \mathrm{i}>\mathrm{j} \geq 1
$$

These results agree with equations (1.5.15) to (1.5.20) of Indra [4].

\section{NUMERICAL RESULTS}

In this section, we present numerical illustrations in order to justify the implementation of analytical results. We have used software MATLAB to develop computational program.

1. The numerical results for the probabilities of exact number of arrivals

(i) by a given time i.e. $\sum_{j=0}^{i} P_{i, j}(t)$

(ii) during busy period i.e. $\sum_{\mathrm{j}=0}^{\mathrm{i}} \mathrm{P}_{\mathrm{i}, \mathrm{j}, \mathrm{B}}(\mathrm{t})$

(iii) and during vacation period i.e. $\sum_{\mathrm{j}=0}^{\mathrm{i}} \mathrm{P}_{\mathrm{i}, \mathrm{j}, \mathrm{V}}(\mathrm{t})$

are computed for different sets of parameter and are summarized in Table - I. The Table - I shows complete agreement with the Table - I of Pegden \& Rosenshine [9] except the columns having probabilities of arrivals during busy period and vacation period.

Table-I is based on the relationship

$\operatorname{Pr}\{i$ arrivals in $(0, t)\}=\frac{\mathrm{e}^{-\lambda t}(\lambda \mathrm{t})^{\mathrm{i}}}{\mathrm{i} !}=\sum_{\mathrm{j}=0}^{\infty} \mathrm{P}_{\mathrm{i}, \mathrm{j}}(\mathrm{t})$

where $P_{i, j}(t)=P_{i, j, V}(t)+P_{i, j, B}(t)\left(1-\delta_{i, j}\right)$. 
Table - I

\begin{tabular}{|l|l|l|l|l|l|l|l|l|l|}
\hline$\lambda$ & $\mu_{\mathrm{B}}$ & $\mu_{\mathrm{V}}$ & $\mathrm{W}$ & $\mathrm{t}$ & $\mathrm{I}$ & $\mathrm{e}^{-\lambda \mathrm{t}}(\lambda \mathrm{t})^{\mathrm{i}}$ & $\sum_{\mathrm{j}=0}^{\mathrm{i}} \mathrm{P}_{\mathrm{i}, \mathrm{B}, \mathrm{B}}(\mathrm{t})$ & $\sum_{\mathrm{j}=0}^{\mathrm{i}} \mathrm{P}_{\mathrm{i}, \mathrm{j}, \mathrm{V}}(\mathrm{t})$ & $\sum_{\mathrm{j}=0}^{\mathrm{i}} \mathrm{P}_{\mathrm{i}, \mathrm{j}}(\mathrm{t})$ \\
\hline 1 & & & & & & & & & \\
\hline 1 & 2 & 1 & 1 & 3 & 3 & 0.224042 & 0.0678183 & 0.1562235 & 0.2240418 \\
\hline 1 & 2 & 1 & 1 & 3 & 5 & 0.100819 & 0.0546483 & 0.0463363 & 0.1008188 \\
\hline 2 & 2 & 1 & 1 & 3 & 1 & 0.014873 & 0.0012179 & 0.0136546 & 0.0148725 \\
\hline 2 & 2 & 1 & 1 & 3 & 3 & 0.089235 & 0.0270118 & 0.0622233 & 0.0892350 \\
\hline 2 & 2 & 1 & 1 & 3 & 5 & 0.160623 & 0.0868008 & 0.0738223 & 0.1606231 \\
\hline 1 & 2 & 1 & 1 & 4 & 1 & 0.073263 & 0.0045651 & 0.0686975 & 0.0732625 \\
\hline 1 & 2 & 1 & 1 & 4 & 3 & 0.195367 & 0.0448534 & 0.1505134 & 0.1953668 \\
\hline 1 & 2 & 1 & 1 & 4 & 5 & 0.156293 & 0.0680836 & 0.0882098 & 0.1562934 \\
\hline 2 & 2 & 1 & 1 & 4 & 1 & 0.002684 & 0.0001672 & 0.0025165 & 0.0026837 \\
\hline 2 & 2 & 1 & 1 & 4 & 3 & 0.028626 & 0.0065721 & 0.0220540 & 0.0286261 \\
\hline 2 & 2 & 1 & 1 & 4 & 5 & 0.091604 & 0.0399038 & 0.0516998 & 0.0916036 \\
\hline 2 & 4 & 2 & 1 & 4 & 5 & 0.091604 & 0.0137116 & 0.0778920 & 0.0916036 \\
\hline 1 & 2 & 1 & 1 & 4 & 4 & 0.195367 & 0.0645246 & 0.1308422 & 0.1953668 \\
\hline 1 & 2 & 1 & 1 & 3 & 6 & 0.050409 & 0.0322999 & 0.0181094 & 0.0504094 \\
\hline 1 & 2 & 1 & 1 & 4 & 4 & 0.195367 & 0.0645246 & 0.1308422 & 0.1953668 \\
\hline 1 & 2 & 1 & 1 & 3 & 6 & 0.050409 & 0.0322999 & 0.0181094 & 0.0504094 \\
\hline
\end{tabular}

2. The departure process from the $\mathrm{M} / \mathrm{M} / 1$ queue has the distribution function $P(j, t)$, the probability that exactly $j$ customers have been served by time $t$. In terms of $P_{i, j}(t)$, we have

$$
P(j, t)=\sum_{i=j}^{\infty} P_{i, j}(t)
$$

$\& \quad P(j, t)=P_{B}(j, t)+P_{V}(j, t)$

where $P_{B}(j, t)=\sum_{i=j}^{\infty} P_{i, j, B}(t) \stackrel{\&}{ } P_{V}(j, t)=\sum_{i=j}^{\infty} P_{i, j, V}(t)$

Tables II, III \& IV show values of $P_{B}(j, t), P_{V}(j, t) \& P(j, t)$ respectively for different values of $t$. Table IV coincides up to six decimal places with table I of Hubbard et al. [3] because they computed 28 values $\&$ we are able to compute only 8 values.

Figs. 2(a)-2(c) display the effect of different values of $\lambda$ on $P_{B}(j, t), P_{V}(j, t) \& P(j, t)$.

Table - II

Values of $\mathrm{P}_{\mathrm{B}}(\mathrm{j}, \mathrm{t})$ for $\lambda=1, \mu_{\mathrm{B}}=4, \mu_{\mathrm{V}}=4 \& \mathrm{w}=1$

\begin{tabular}{|c|c|c|c|c|c|}
\hline $\mathbf{j}$ & $\mathbf{t}=\mathbf{1}$ & $\mathbf{t}=\mathbf{3}$ & $\mathbf{t}=\mathbf{5}$ & $\mathbf{t}=\mathbf{7}$ & $\mathbf{t}=\mathbf{1 0}$ \\
\hline 0 & 0.0 & 0.0 & 0.0 & 0.0 & 0.0 \\
\hline 1 & 0.02623 & 0.00414 & 0.00056 & 0.00007 & 0.00000378 \\
\hline 2 & 0.02030 & 0.01200 & 0.00274 & 0.00052 & 0.00003742 \\
\hline
\end{tabular}

\begin{tabular}{|l|l|l|l|l|l|}
\hline 3 & 0.00703 & 0.01723 & 0.00670 & 0.00180 & 0.00018476 \\
\hline 4 & 0.00144 & 0.01624 & 0.01082 & 0.00410 & 0.00060433 \\
\hline 5 & 0.00019 & 0.01113 & 0.01280 & 0.00685 & 0.00145219 \\
\hline 6 & 0.00001 & 0.00562 & 0.01127 & 0.00854 & 0.00259984 \\
\hline 7 & 0.00000 & 0.00179 & 0.00631 & 0.00677 & 0.00296147 \\
\hline
\end{tabular}

Table - III

Values of $\mathrm{P}_{\mathrm{V}}(\mathrm{j}, \mathrm{t})$ for $\lambda=1, \mu_{\mathrm{B}}=4, \mu_{\mathrm{V}}=4 \& \mathrm{w}=1$

\begin{tabular}{|c|c|c|c|c|c|}
\hline $\mathbf{j}$ & $\mathbf{t}=\mathbf{1}$ & $\mathbf{t}=\mathbf{3}$ & $\mathbf{t}=\mathbf{5}$ & $\mathbf{t}=\mathbf{7}$ & $\mathbf{t}=\mathbf{1 0}$ \\
\hline 0 & 0.45816 & 0.06223 & 0.00842 & 0.00113 & 0.0000567 \\
\hline 1 & 0.34083 & 0.17051 & 0.03992 & 0.00768 & 0.0005527 \\
\hline 2 & 0.11726 & 0.23158 & 0.09433 & 0.02585 & 0.0026898 \\
\hline 3 & 0.02458 & 0.20747 & 0.14811 & 0.05789 & 0.0087187 \\
\hline 4 & 0.00350 & 0.13755 & 0.17363 & 0.09699 & 0.0211590 \\
\hline 5 & 0.00036 & 0.07156 & 0.16145 & 0.12915 & 0.0408493 \\
\hline 6 & 0.00002 & 0.02982 & 0.12163 & 0.13957 & 0.0640349 \\
\hline 7 & 0.00001 & 0.00913 & 0.06789 & 0.11175 & 0.0743153 \\
\hline
\end{tabular}

Table - IV

Values of $P(j, t)$ for $\lambda=1, \mu_{B}=4, \mu_{V}=4 \& w=1$

\begin{tabular}{|c|c|c|c|c|c|}
\hline $\mathbf{j}$ & $\mathbf{t}=\mathbf{1}$ & $\mathbf{t}=\mathbf{3}$ & $\mathbf{t}=\mathbf{5}$ & $\mathbf{t}=\mathbf{7}$ & $\mathbf{t}=\mathbf{1 0}$ \\
\hline 0 & 0.48440 & 0.066379 & 0.00898 & 0.00121 & 0.00006 \\
\hline 1 & 0.36114 & 0.182525 & 0.04267 & 0.00820 & 0.00059 \\
\hline 2 & 0.12430 & 0.248821 & 0.10104 & 0.02765 & 0.00287 \\
\hline 3 & 0.02602 & 0.223725 & 0.15893 & 0.06199 & 0.000932 \\
\hline 4 & 0.00370 & 0.148688 & 0.18643 & 0.10385 & 0.022611 \\
\hline 5 & 0.00038 & 0.077192 & 0.17272 & 0.13769 & 0.043449 \\
\hline 6 & 0.00002 & 0.031621 & 0.12794 & 0.14634 & 0.066996 \\
\hline 7 & 0.00000 & 0.009139 & 0.06789 & 0.11175 & 0.074315 \\
\hline Total & $\mathbf{0 . 9 9 9 9 8}$ & $\mathbf{0 . 9 8 8 0 9 5}$ & $\mathbf{0 . 8 6 6 6 2}$ & $\mathbf{0 . 5 9 8 7 1}$ & $\mathbf{0 . 2 2 0 2 2 0}$ \\
\hline
\end{tabular}

3. The probability of exactly $n$ customers in the system at time $\mathrm{t}$, denoted by $\mathrm{P}(\mathrm{n}, \mathrm{t})$ can be expressed in terms of

$P_{i, j}(t)$ as

$P(n, t)=\sum_{j=0}^{\infty} P_{j+n, j}(t) \& \quad P(n, t)=P_{B}(n, t)+P_{V}(n, t)$

Where

$P_{B}(n, t)=\sum_{j=0}^{\infty} P_{j+n, j, B}(t) \quad \& \quad P_{V}(n, t)=\sum_{j=0}^{\infty} P_{j+n, j, V}(t)$

Values of $\mathrm{P}_{\mathrm{B}}(\mathrm{n}, \mathrm{t}), \mathrm{P}_{\mathrm{V}}(\mathrm{n}, \mathrm{t}), \quad$ and $\mathrm{P}(\mathrm{n}, \mathrm{t}) \quad$ with parameters $\lambda=1, \quad \mu_{\mathrm{B}}=2, \quad \mathrm{~W}=1$ and $\mu_{\mathrm{V}}=1$ for different values of $t$ are shown in the following tables.

Figs. 3(a)-3(c) depict the effect of different values of $\lambda$ on $\mathrm{P}_{\mathrm{B}}(\mathrm{n}, \mathrm{t}), \mathrm{P}_{\mathrm{V}}(\mathrm{n}, \mathrm{t}) \& \mathrm{P}(\mathrm{n}, \mathrm{t})$.

Table $\mathrm{V}$ for $\mathrm{P}_{\mathrm{B}}(\mathrm{n}, \mathrm{t})$

\begin{tabular}{|c|c|c|c|}
\hline $\mathbf{N}$ & $\mathbf{t}=\mathbf{1}$ & $\mathbf{t}=\mathbf{3}$ & $\mathbf{t = 5}$ \\
\hline 0 & 0.0 & 0.0 & 0.0 \\
\hline 1 & 0.082290012 & 0.116579206 & 0.114237580 \\
\hline 2 & 0.041450927 & 0.093201930 & 0.093478491 \\
\hline 3 & 0.012999844 & 0.052851773 & 0.054290691 \\
\hline 4 & 0.003066883 & 0.024798463 & 0.025659204 \\
\hline
\end{tabular}




\begin{tabular}{|l|l|l|l|}
\hline 5 & 0.000584727 & 0.009916438 & 0.009929580 \\
\hline 6 & 0.000093697 & 0.003299066 & 0.003000457 \\
\hline 7 & 0.000012835 & 0.000829619 & 0.000633436 \\
\hline 8 & 0.000001365 & 0.000118837 & 0.000070241 \\
\hline
\end{tabular}

Table VI for $\mathrm{P}_{\mathrm{V}}(\mathrm{n}, \mathrm{t})$

\begin{tabular}{|c|c|c|c|}
\hline $\mathbf{N}$ & $\mathbf{t = 1}$ & $\mathbf{t}=\mathbf{3}$ & $\mathbf{t}=\mathbf{5}$ \\
\hline 0 & 0.551515356 & 0.421382585 & 0.392520270 \\
\hline 1 & 0.215830892 & 0.166496430 & 0.149569409 \\
\hline 2 & 0.069400881 & 0.065943899 & 0.056541479 \\
\hline 3 & 0.018069955 & 0.025826719 & 0.021010086 \\
\hline 4 & 0.003870518 & 0.009800191 & 0.007533957 \\
\hline 5 & 0.000697602 & 0.003510927 & 0.002516171 \\
\hline 6 & 0.000107746 & 0.001138796 & 0.000732218 \\
\hline 7 & 0.000014475 & 0.000303332 & 0.000163226 \\
\hline 8 & 0.000001576 & 0.000049791 & 0.000020523 \\
\hline
\end{tabular}

Table VII for $\mathrm{P}(\mathrm{n}, \mathrm{t})$

\begin{tabular}{|c|c|c|c|}
\hline $\mathbf{N}$ & $\mathbf{t}=\mathbf{1}$ & $\mathbf{t}=\mathbf{3}$ & $\mathbf{t}=\mathbf{5}$ \\
\hline 0 & 0.551515356 & 0.421382585 & 0.392520270 \\
\hline 1 & 0.298110904 & 0.283075636 & 0.263806989 \\
\hline 2 & 0.110851808 & 0.159145829 & 0.150019970 \\
\hline 3 & 0.031069799 & 0.078678492 & 0.075300777 \\
\hline 4 & 0.006937401 & 0.034598654 & 0.033193161 \\
\hline 5 & 0.001282329 & 0.013427365 & 0.012445751 \\
\hline 6 & 0.000201443 & 0.004437862 & 0.003732675 \\
\hline 7 & 0.000027310 & 0.001132951 & 0.000796662 \\
\hline 8 & 0.000002940 & 0.000168628 & 0.000090764 \\
\hline Total & $\mathbf{0 . 9 9 9 9 9 9 2 9 0}$ & $\mathbf{0 . 9 9 6 0 4 8 0 0 2}$ & $\mathbf{0 . 9 3 1 9 0 7 0 1 9}$ \\
\hline
\end{tabular}

4. The waiting time distribution for a customer can be derived as $\mathbf{P}(W>\tau \mid t)$, the probability that a customer waits more than $\tau$ time units in the system, given that the customer arrives at time $t$.

$\mathrm{P}(\mathrm{W}>\tau \mid \mathrm{t})=\sum_{\mathrm{n}=0}^{\infty} \mathrm{P}($ number of services by time $\tau<\mathrm{n}+1)$

$$
\mathrm{P}_{\mathrm{n}}(\mathrm{t})=\mathrm{e}^{-\mu \tau} \sum_{\mathrm{n}=0}^{\infty} \sum_{\mathrm{s}=0}^{\mathrm{n}} \frac{(\mu \tau)^{\mathrm{s}}}{\mathrm{s} !} \mathrm{P}_{\mathrm{n}}(\mathrm{t})
$$

The cumulative distribution for the sojourn time in the system is

$$
\mathrm{P}(\mathrm{W} \leq \tau \mid \mathrm{t})=1-\mathrm{e}^{-\mu \tau} \sum_{\mathrm{n}=0}^{\infty} \sum_{\mathrm{s}=0}^{\mathrm{n}} \frac{(\mu \tau)^{\mathrm{s}}}{\mathrm{s} !} \mathrm{P}_{\mathrm{n}}(\mathrm{t})
$$

Table - VIII is based on the above relationships and the graphical representation for waiting time and cumulative distribution for the sojourn time in the system has also been shown in the fig -4 and 5 respectively.

Table-VIII

For $\lambda=1, \mu_{\mathrm{V}}=1, \mu_{\mathrm{B}}=2, \mathrm{w}=1$

\begin{tabular}{|l|c|}
\hline$\tau$ & $\mathbf{P}(\mathbf{W}>\tau \mid \mathbf{t})$ \\
\hline 1 & 0.66237681368507 \\
\hline 2 & 0.26611626185091 \\
\hline 3 & 0.07975130381442 \\
\hline 4 & 0.02106267584575 \\
\hline 5 & 0.00516058582580 \\
\hline 6 & 0.00119987862663 \\
\hline
\end{tabular}

5. The system utilization, i.e. the fraction of time the server is busy until time $t$ can also be expressed in terms of $P_{i, j}(t)$.

Thus the fraction of the time the system is empty and consequently the server is on working vacation is

$$
\mathrm{U}_{\mathrm{v}}(\mathrm{t})=\sum_{\mathrm{i}=0}^{\infty} \sum_{\mathrm{j}=0}^{\mathrm{i}} \mathrm{P}_{\mathrm{i}, \mathrm{j}, \mathrm{V}}(\mathrm{t})
$$

and the fraction of time that the system is non-empty and hence the server utilized is

$$
\mathrm{U}_{\mathrm{B}}(\mathrm{t})=\sum_{\mathrm{i}=0}^{\infty} \sum_{\mathrm{j}=0}^{\mathrm{i}} \mathrm{P}_{\mathrm{i}, \mathrm{j}, \mathrm{B}}(\mathrm{t})
$$

also total utilization time of server is given by

$$
\mathrm{U}(\mathrm{t})=\mathrm{U}_{\mathrm{B}}(\mathrm{t})+\mathrm{U}_{\mathrm{V}}(\mathrm{t})
$$

Table - IX is based on the above relationships and the graphical representation has also been shown in the figs. 6(a), 6(b) and 6(c).

Table-IX

For $\lambda=1, \mu_{\mathrm{V}}=1, \mu_{\mathrm{B}}=2, \mathrm{w}=1$

\begin{tabular}{|c|c|l|l|}
\hline $\mathrm{t}$ & $\begin{array}{l}\text { Utilization time } \\
\text { during busy period } \\
\mathrm{U}_{\mathrm{B}}(\mathrm{t})\end{array}$ & $\begin{array}{l}\text { Utilization time } \\
\text { during working } \\
\text { vacation period } \\
\mathrm{U}_{\mathrm{V}}(\mathrm{t})\end{array}$ & $\begin{array}{l}\text { Total Utilization time } \\
\mathrm{U}(\mathrm{t})\end{array}$ \\
\hline 1 & 0.14044480842 & 0.859471950429 & 0.9999167588507 \\
\hline 2 & 0.24487805973 & 0.750588134738 & 0.9954661944737 \\
\hline 3 & 0.27965057813 & 0.686840886556 & 0.9664914646911 \\
\hline 4 & 0.25857017114 & 0.630755850451 & 0.8893260215974 \\
\hline 5 & 0.20603419772 & 0.556149265245 & 0.7621834629729 \\
\hline
\end{tabular}
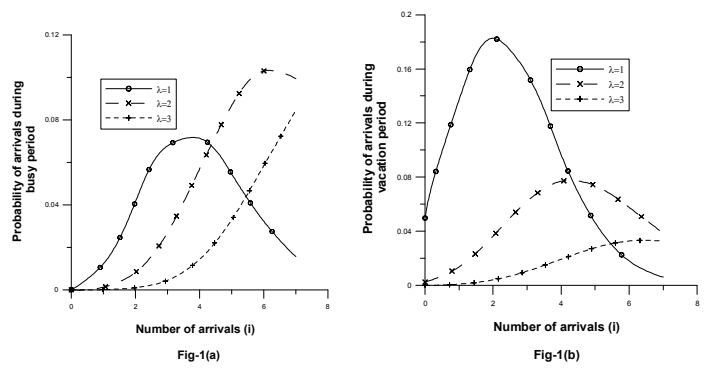

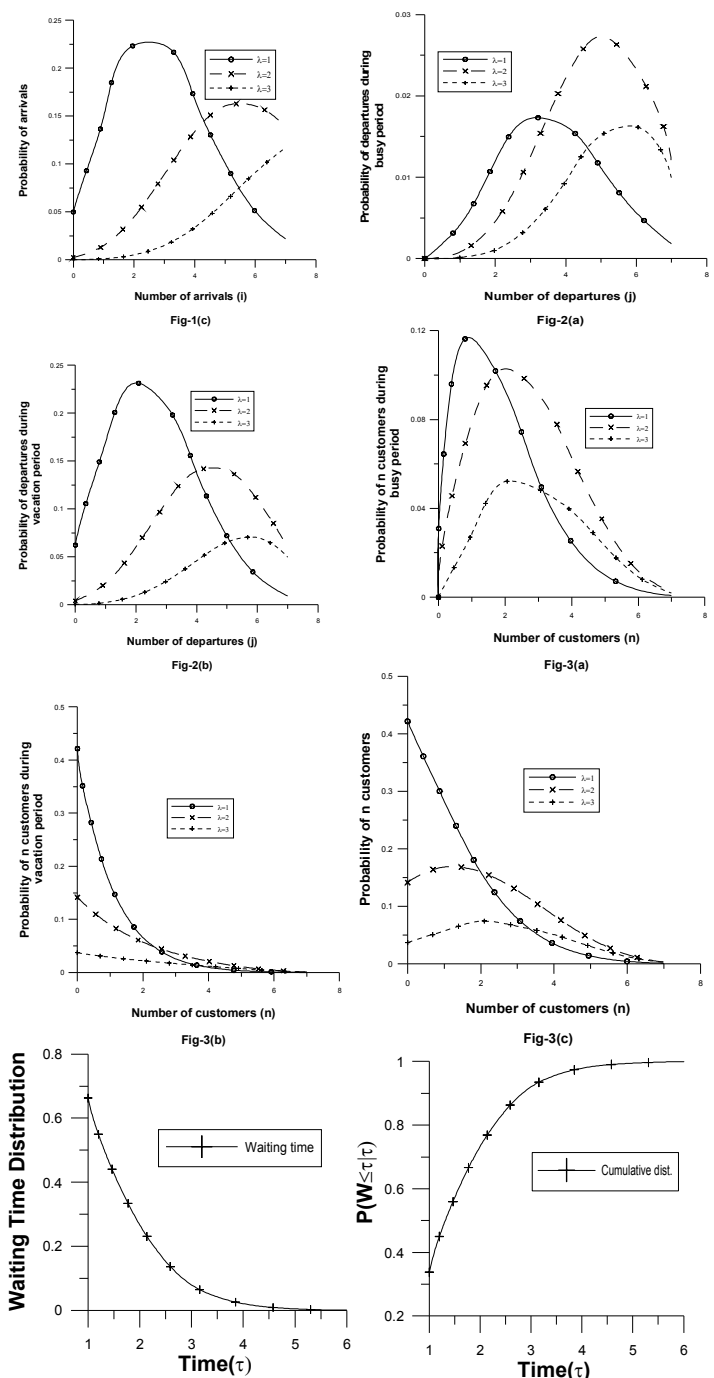

Fig-3(c)

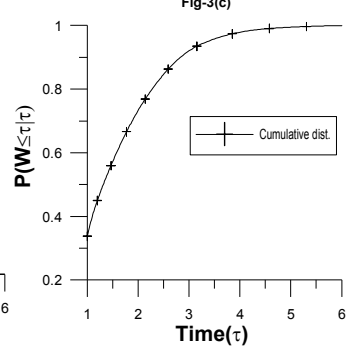

Fig - 4
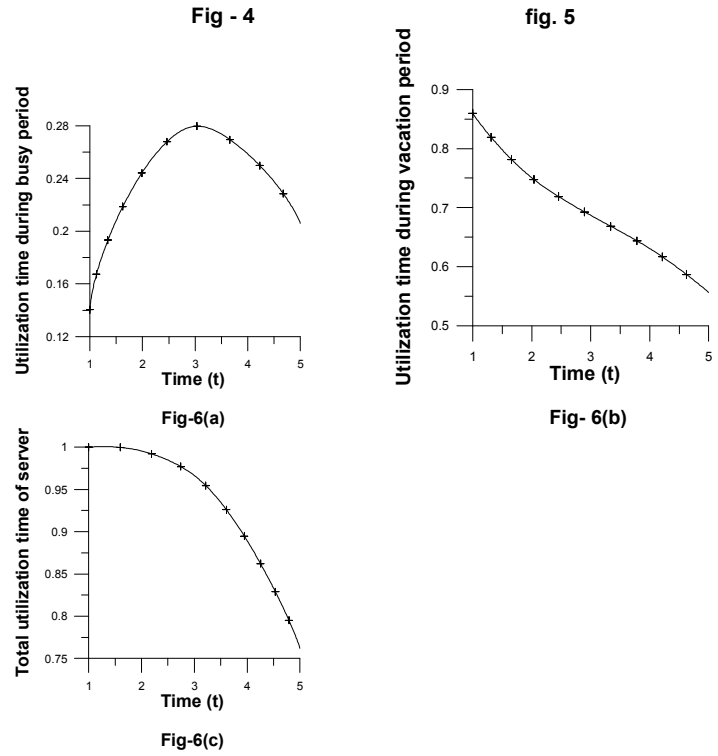

\section{CONCLUSION}

The two-dimensional state $\mathrm{M} / \mathrm{G} / 1$ queueing system with working vacation has been investigated. The applications of working vacation in manufacturing process, computer and communication networks are well established. Several performance measures are derived numerically by using Matlab programming. The present investigation can be extended by incorporating bulk input/service. However numerical technique based on matrix-geometric approach can be implemented.

\section{ACKNOWLEDGEMENT}

We are grateful to the Chairperson, Department of Statistics \& Operational Research, Kurukshetra University, Kurukshetra for providing necessary facilities and showing keen interest in this work.

\section{REFERENCES}

[1] Baba, Y., (2005), Analysis of a GI/M/1 queue with multiple working vacations Oper. Res. Lett. 33, 201-209.

[2] Banik, A.D., Gupta, U.C., Pathak, S.S. (2007), On the $\mathrm{GI} / \mathrm{M} / 1 / \mathrm{N}$ queue with multiple working vacations analytic analysis and computation Appl. Math. Modell. 31(9), 1701-1710.

[3] Hubbard J.R., Pegden C.D., and Rosenshine M., (1986), The departure process for the $\mathrm{M} / \mathrm{M} / 1$ queue, Journal of Applied Probability, Vol. 23, No. 1, (Mar.,1986), pp.249-255.

[4] Indra, (1994), Some two-state single server queueing models with vacation or latest arrival run, Ph.D. thesis, Kurukshetra University, Kurukshetra.

[5] Indra and Ruchi, (2009), Transient Analysis of Two-Dimensional M/M/1 Queueing System with working vacations, Journal of Mathematics and System Science, Vol. 5, No. 2, (Dec. 2009) pp. 110-128.

[6] Kim, J.D., Choi, D.W., Chae, K.C, (2003), Analysis of queue-length distribution of the M/G/1 queue with working vacations In: Hawaii International Conference on Statistics and Related Fields, Hanolulu, Hawaii, June 5-8.

[7] Li, Ji-hong, and Tian, Nai-shuo (2006), Analysis of the discrete time $\mathrm{Geo} / \mathrm{Geo} / 1$ queue with single working vacation QTQM, 5, 77-89.

[8] Liu, Wen-yuan, Xu, Xiu-li, and Tian, Nai-shuo (2006), Stochastic decomposition in the $M / M / 1$ queue with working vacation Operational Research letters, 35, 595-600.

[9] Pegden, C.D. and Rosenshine, M. (1982), Some new results for the M/M/1 queue, Mgt Sci 28, 821-828 (1982).

[10] Servi, L.D. and Finn, S.G., (2002), M/M/1 queues with working vacations (M/M/1/WV), Performance Evaluation, Vol. 50, pp 41-52.

[11] Wu, D., Takagi, H., (2002), M/G/1 queue with multiple working vacations. Perform. Eval. 63(7), 654-681. 